Claves. Revista de Historia, Vol. $3, \mathrm{~N}^{\mathrm{o}} 5$

Montevideo, julio - diciembre 2017

(pp. 155-183) ISSN 2393-6584

\title{
Los archivos represivos y el debate sobre los criterios para su mejor utilización como instrumento de justicia y de derechos ${ }^{1}$
}

\author{
Repressive files: \\ A debate on the criteria for their best use \\ as an instrument for justice and rights
}

Gerardo Caetano ${ }^{2}$

Universidad de la República, Uruguay

Recibido: 11/09/2017

Aceptado: 25/10/2017

Resumen: El artículo analiza el tratamiento de los archivos represivos como tópico central de los procesos de investigación de las violaciones a los derechos humanos. A partir de ciertas características generales toma como referencia la situación uruguaya hasta el año 2010, aborda el tema desde una visión global registrando las principales pautas de la experiencia internacional reciente y procura indagar en torno a los sistemáticos obstáculos interpuestos para la plena consecución de verdad y justicia, de sanción a los culpables y reparación de las víctimas.

Palabras clave: archivos represivos, justicia, derechos humanos, Uruguay

\footnotetext{
${ }^{1}$ Los debates recientes originados en torno al manejo del llamado "Archivo Berruti" (localizado en dependencias del Ministerio de Defensa Nacional en el año 2006, cuando la titular de la cartera era la Dra. Azucena Berruti, trasladado luego al Archivo General de la Nación en 2009 y puesto bajo reserva por 15 años en el 2011, de acuerdo a los criterios establecidos en la legislación vigente) y del llamado "Archivo Castiglioni" (con documentación localizada en el domicilio particular del militar fallecido Elmar Castiglioni, transferida luego a dependencias del Poder Judicial y que ha originado la conformación de una Comisión Investigadora Parlamentaria) imponen volver a debatir estos temas, en una materia en la que el rezago del país se mantiene ostensible. El manejo periodístico que ha tenido esta documentación, en especial por el periodista Samuel Blixen y el semanario "Brecha", que finalmente ha culminado con la publicación de aproximadamente 14.000 de los cerca de tres millones de documentos que se presume contiene el primer "Archivo" mencionado, ha profundizado el debate, del que han participado, como referiremos más adelante, muchos historiadores dedicados al campo. Ante distintas solicitudes de colegas he resuelto finalmente volver a publicar este artículo que fundamentalmente ha circulado en el exterior del país, en revistas académicas especializadas. La dimensión pública y la relevancia del debate en curso han impulsado esta decisión, con el compromiso de avanzar en forma directa sobre los acontecimientos más recientes en un trabajo próximo.

${ }^{2}$ Historiador y politólogo.
} 
Abstract: The article analyzes the treatment of repressive files as a central topic in the investigation processes of human rights violations. Based on certain generic characteristics, the present work takes as a reference the Uruguayan situation until 2010, addressing the issue from a global perspective, recording the main standards of recent international experience, and seeking to research on the systematic obstacles interposed for the attainment of truth and justice, penalties for the guilty, and reparation for the victims.

Key words: repressive files, justice, human rights, Uruguay

\section{Introducción}

En verdad, pese a su reciente creación y utilización, el concepto de "justicia transicional" ha sido y es sometido a frecuente debate, tanto en el campo académico como en el de la política, el derecho o el de la acción en defensa de los derechos humanos. En primer término, su propia definición genera controversias. Hay quienes incluso le han negado legitimidad, ya que asocian el concepto a la imposición forzosa de concesiones gravosas en el plano de la búsqueda de la verdad y la justicia, en el marco de los azarosos procesos de transición que siguen a las dictaduras o a períodos de conflicto.

Más allá de las controversias, distintos autores desde perspectivas incluso disímiles, han coincidido en registrar un cúmulo de rasgos identificatorios de los procesos de "justicia transicional": i) estos se asocian con proyectos de cambio político profundo en tanto su despliegue apunta a enfrentar los múltiples legados de pasados traumáticos, signados por violencia y por violaciones flagrantes a los derechos humanos; ii) aunque están demandados por exigencias y lógicas contrapuestas debido al contraste entre las perspectivas involucradas, en su tramitación se busca alterar las pautas dominantes de la situación heredada para volcarla a favor de las víctimas, en procura de un desplazamiento efectivo de los victimarios en relación a toda situación de poder; iii) si bien su objetivo central es consolidar el retorno a situaciones de normalidad democrática y a estados de paz y de vigencia del derecho, quienes impulsan y conducen estos procesos descubren muy a menudo la necesidad de afrontar compromisos radicales y de no eludir conflictos difíciles para afirmar de manera 
genuina la consecución de los valores anhelados (justicia, verdad, memoria y reparación); iv) aunque la conducción de estos procesos está tensionada por las características locales y específicas de cada transición, existe una creciente conciencia a favor de adecuar las soluciones en cada caso a las recomendaciones jurídicas y políticas provenientes de los consensos de mayor exigencia logrados a nivel internacional, en el marco de lo que ha venido afirmándose como el Derecho Internacional de los Derechos Humanos; iv) si bien su propio nombre refiere a la prioridad del tema justicia, sus objetivos resultan más generales y remiten a la necesidad de instrumentos variados (desde acciones propiamente judiciales a sanciones no criminales, desde "comisiones de verdad" a un amplio abanico de medidas reparatorias y a construcción de nueva institucionalidad en distintos campos). 34

En este artículo se trabajará a propósito de un tópico con frecuencia olvidado y al que asignamos una relevancia especial dentro de lo que comúnmente se denominan "políticas de memoria": el tratamiento de los archivos represivos como tópico central de los procesos de investigación de las violaciones a los derechos humanos. En primer lugar remitiremos a ciertas características generales del tema, tomando como referencia ilustrativa la situación uruguaya en la materia básicamente hasta el 2010. A continuación, el tema se abordará desde una visión más global, registrando las principales pautas orientadoras que indica la experiencia internacional reciente sobre el tema. A partir del análisis de estos temas, se procurará indagar en torno a un aspecto que ha sido clave en los sistemáticos obstáculos interpuestos para la

\footnotetext{
3 Para visiones diversas en torno al tema de los procesos de "justicia transicional", cfr.: Ambos-MalarinoElsner (eds.), 2009; Aguilar Fernández, 2008; Elster, 2006; Rottberg, 2005; Decker y Keppler, 2004; Vinjamuri y Snyder, 2004; Teitel, 2003; entre otros.

${ }^{4}$ En tiempos recientes, la temática de los criterios de la "Justicia transicional" ha recobrado mucho vigor, en especial a partir de las experiencias que en la materia se han verificado en el proceso de paz en Colombia entre el gobierno del presidente Juan Manuel Santos y el grupo guerrillero denominado Fuerzas Armadas Revolucionarias de Colombia (FARC), culminado en 2017. Al respecto cfr. especialmente: i) Comunicado Conjunto. La Habana, marzo 7, 2015. (Documento que reúne doce ensayos de historiadores con visiones plurales de historiadores colombianos, 809 páginas); ii) Informe conjunto de la mesa de conversaciones de Paz entre el Gobierno de la República de Colombia y las Fuerzas Armadas Revolucionarias de Colombia-Ejército del Pueblo, (FARC-EP): Avances de las discusión del Punto 5: Comisión para el esclarecimiento de la verdad, la convivencia y la no repetición. 4 de junio de 2015; y iii) Comunicado Conjunto $\mathrm{N}^{\circ}$ 64. Acuerdo sobre las víctimas del conflicto. Acuerdo sobre las Víctimas del Conflicto "Sistema Integral de Verdad, Justicia, Reparación y No Repetición”, incluyendo la Jurisdicción Especial para la Paz; y Compromiso sobre Derechos Humanos. La Habana, 15 de diciembre de 2015.
} 
plena consecución de verdad y justicia, de sanción a los culpables y reparación de las víctimas.

En esta dirección, en el artículo que sigue se intenta dar respuesta a la siguiente pregunta: ¿Por qué los imperativos de memoria, verdad, justicia y reparación -inherentes a todo proceso de justicia transicional- no han sido suficientes en América Latina para generar la aplicación de criterios de efectiva rigurosidad en el tratamiento de los archivos de la represión? En la exploración en torno a esta indagatoria central debe incorporarse el reto de explicar, en la misma dirección, por qué los Estados nacionales del continente han eludido con frecuencia la asunción plena del reclamo de justicia y verdad como una de las principales obligaciones imperantes en el Derecho Internacional de los Derechos Humanos. En este sentido, vale la pena recordar que la Corte Interamericana de Derechos Humanos ha establecido en varias de sus sentencias la obligación de los Estados de investigar a fondo las violaciones a los derechos humanos y de sancionar a los autores intelectuales y materiales de las mismas. Del mismo modo, en la jurisprudencia de la misma Corte puede encontrarse un consistente desarrollo en torno al concepto de reparación integral a las víctimas, el que entre otras cosas supone la consagración de los derechos a la verdad y a la memoria como vía ineludible para que el Estado afinque soportes efectivos para la no repetición de los actos violatorios de derechos humanos.

El argumento central que se desarrollará en las páginas que siguen apunta a destacar que una de las claves explicativas más relevantes para entender por qué los Estados no han atendido las estrategias esenciales en el tratamiento de los archivos represivos radica en que estos temas no han sido entendidos ni encarados desde la perspectiva radical de una cuestión fundamental de derechos humanos. Lejos de esta exigencia, los Estados latinoamericanos han considerado estos asuntos esenciales de los procesos de justicia transicional desde una perspectiva elusiva y secundaria, de modo coherente con visiones "posibilistas" y en extremo "minimalistas" de la construcción de las democracias post dictatoriales. La hipótesis central del artículo se orienta a fundamentar la idea de que sólo desde una lógica radical de derechos humanos y desde la asunción plena de las exigencias del Derecho 
Internacional contemporáneo en la materia es que se pueden construir a nuestro juicio políticas públicas efectivas en torno al tratamiento de los archivos represivos, núcleo indispensable de cualquier iniciativa fundada para concretar los imperativos de la verdad, la justicia, la memoria y la reparación.

\section{Nuevos enfoques y exigencias para los procesos de "justicia transicional"}

Como lo prueban estudios recientes, el análisis atento y profundo de estos procesos de búsqueda de justicia y verdad luego de dictaduras y de pasados traumáticos a menudo tiene la virtud complementaria de agregar evidencia respecto a la debilidad e inconsistencia de muchos lugares comunes que legó la llamada "literatura de las transiciones," en boga hace dos décadas. En esta dirección, por ejemplo, en varios de sus últimos trabajos, Kathryn Sikkink 5 ha podido abogar con persuasividad acerca de cómo el estudio de lo que ha llamado "la cascada de justicia y el impacto de los juicios de derechos humanos" desplegados en las últimas décadas en América Latina no sólo ha cuestionado las principales hipótesis manejadas por algunos de los estudiosos más connotados de las transiciones, sino que ha podido fundar la conclusión de que la realización y profundización de los principios de justicia y verdad no ha "socavado la democracia ni ha llevado a un aumento de las violaciones de derechos humanos o de los conflictos" en el continente. ${ }^{6}$

Luego de sistematizar algunos de los argumentos más aceptados (y a la vez más polémicos) de la revisión de la "literatura de las transiciones", Sikkink y Booth Walling en el trabajo antes referido realizan un pormenorizado estudio sobre la evolución específica en América Latina de los distintos mecanismos de "justicia de transición" ("comisiones de verdad", procesos judiciales, juicios internos, en el extranjero, mixtos, etc.). Los datos y evidencias obtenidos en su investigación permiten sustentar conclusiones fuertes:

"Nuestra investigación -señalan las autoras- cuestiona algunos supuestos básicos de la literatura sobre la transición, incluyendo que los juicios socavan la democracia, que las decisiones sobre los juicios deben ser tomadas a principios del

\footnotetext{
${ }^{5}$ SIKKINK, Kathryn; BOOTH-WALLING, Carrie, (2008); SIKKINK (2005); SIKKINK y LUTZ, Ellen, (2000).

${ }^{6}$ SIKKINK, Kathryn; BOOTH-WALLING, p. 16.
} 
período posterior a la transición pues de lo contrario no serán posibles y que las opciones sobre amnistías y juicios que se hagan en el período posterior a la transición probablemente serán estables y duraderas.” 7

De manera particular, los resultados de la investigación habilitan a sus autoras a ser especialmente categóricas al desmentir la reiteración de argumentos "pesimistas" por parte de quienes, desde "una nueva literatura de las relaciones internacionales", "repiten las mismas afirmaciones y añaden hipótesis aún más fuertes sobre los peligros de los juicios.”

\begin{abstract}
"Nuestros elementos de prueba -advierten en este sentido- mostraron que los propulsores de los juicios en América Latina no están promoviendo atrocidades sin darse cuenta; que los juicios no aumentan las violaciones a los derechos humanos, no exacerban los conflictos ni representan una amenaza para la democracia; y que no es posible demostrar que las amnistías sirvan para impedir futuras violaciones a los derechos humanos." 8
\end{abstract}

Al final de su artículo, Sikkink y Booth Walling perfilan una nueva perspectiva conceptual en torno a cómo proyectar los desafíos de los procesos de "justicia transicional" que concita nuestro pleno acuerdo:

\begin{abstract}
"ha llegado el momento de dejar atrás las falsas dicotomías y comenzar un debate más matizado sobre la justicia de transición. Las opciones no son entre verdad o justicia, entre juicios o democracia, ni entre idealistas o pragmatistas. En cambio, es mucho más interesante examinar bajo qué condiciones es posible que los juicios contribuyan a mejorar los derechos humanos y reforzar los sistemas de Estado de derecho, o qué secuencia o combinación sensata de mecanismos de justicia de transición puede servir para construir la democracia y resolver los conflictos." 9
\end{abstract}

Esta nueva perspectiva conceptual le otorga a los procesos de "justicia transicional" un nuevo sentido de horizonte de futuro, desde el que la faena insoslayable de intentar la mejor elaboración y sutura de los pasados traumáticos se combina en forma necesaria con rediseños institucionales y reformas sustantivas en varias políticas públicas, en el marco de acciones orientadas a evitar en forma concreta y perdurable la repetición de lo padecido. En esa tarea de construcción institucional y de prospectiva cabe un amplio espectro de temas, como se advertirá a continuación.

\footnotetext{
7 Ibídem, p. 35.

${ }^{8}$ Ibídem, p. 36.

${ }^{9}$ Ibídem, p. 37
} 


\section{Los archivos represivos de la dictadura uruguaya: entre expurgos, hallazgos parciales y desapariciones persistentes}

Como se señala en la parte introductoria del Tomo I de la Investigación Histórica sobre Detenidos Desaparecidos. En cumplimiento del Artículo $4^{\circ}$ de la Ley 15.848, el equipo de historiadores que trabajó en la misma pudo relevar diecinueve archivos vinculados en forma directa o indirecta con la represión durante la dictadura, la mayoría nacionales y algunos extranjeros..$^{10}$ En esa labor se pudo constatar la situación preocupante que exhibía la mayoría de esos archivos en el país, en especial aquellos que se encuentran hasta el presente en la órbita del Estado, con algunas honrosas excepciones justamente mencionadas en la publicación señalada y en otros estudios más recientes. ${ }^{11}$

En la consulta de los archivos nacionales, más allá de la muy buena disposición de algunos funcionarios, se pudo confirmar en efecto un cúmulo de insuficiencias e irregularidades. La más grave de ellas tenía que ver con la desaparición de parte de los repositorios, en algunos casos como consecuencia del cumplimiento directo de resoluciones formales e informales de las autoridades de la dictadura en los últimos meses del régimen. Tal fue el caso, por ejemplo, de la resolución oficial del Ministerio de Relaciones Exteriores fechada el 31 de octubre de 1984, por la que se disponía "la destrucción de documentos en desuso y carentes de interés que hayan sido microfilmados", sin precisión alguna sobre sus contenidos. ${ }^{12}$ Otro caso probado de desaparición de documentos pudo ser verificado en el Poder Legislativo: de acuerdo al testimonio del reconocido funcionario parlamentario Luis María Paravís, las grabaciones dispuestas por la Comisión Investigadora Parlamentaria sobre las actividades del grupo paramilitar denominado "Escuadrón de la Muerte"

\footnotetext{
10 Cfr. RICO, Álvaro (coord.), “Investigación Histórica sobre Detenidos Desaparecidos. 5 Tomos. Montevideo, IMPO, 2007, pp. 11 y ss.

${ }^{11}$ Vania Markarian ha analizado también de manera particular los casos de los archivos de los Ministerios de Relaciones Exteriores, Interior (con especial mención a los repositorios que obran en la Dirección Nacional de Información e Inteligencia) y Defensa Nacional. Cfr. MARKARIAN, Vania, 2009.

${ }^{12}$ Cfr. URUGUAY, Ministerio de Relaciones Exteriores, Decreto 484/984, 31 de octubre de 1984. Diario Oficial, $\mathrm{N}^{\circ}$ 21.861. Registro Nacional de Leyes y Decretos. Tomo 1, Semestre 2, Año 1984, p. 1000. Otro ejemplo sobre este particular fue el decreto 512/984, 15 de noviembre de 1984, por el que se facultaba en este caso al Ministerio de Defensa Nacional "para disponer la destrucción de documentos archivados que estén microfilmados y cuyos originales no ofrezcan interés alguno”. Ambos documentos pueden consultarse en "Investigación Histórica sobre Detenidos Desaparecidos... etc. ob. cit., Tomo IV, Anexo Sección 8, "Sobre destrucción de Archivos de la dictadura", pp. 805 y ss.
} 
(comisión que funcionó durante el año 1972 y que pudo recabar y recopilar los testimonios de las personas que presentaron declaración ante la misma) fueron requisadas en diciembre de 1973 por efectivos militares, desconociéndose hasta el día de hoy su paradero. ${ }^{13}$

Asimismo, como consta también en el Informe citado, se han encontrado en las investigaciones realizadas evidencias contundentes sobre la existencia durante la dictadura uruguaya de varios archivos represivos de decisiva importancia (como los del Servicio de Inteligencia de Defensa, SID, o los de las dependencias del Organo Coordinador de Actividades Antisubversivas, OCOA), radicados entonces en la órbita del Ministerio de Defensa y en el seno de las Fuerzas Armadas, sobre cuyos contenidos poco se ha podido avanzar. Una excepción en la materia, como se ha adelantado al inicio del artículo y se profundizará más adelante, ha sido el hallazgo en el año 2006 del llamado "Archivo Berruti" y más cerca en el tiempo la documentación requisada e incautada por la Justicia en el 2015 en el domicilio del coronel fallecido Elmar Castiglioni. ${ }^{14}$

Aquí se encuentra un primer desafío ineludible que hace a la impostergable ubicación de los archivos de la represión existentes durante la dictadura, y como podemos saber hoy también operativos en democracia ${ }^{15}$, a los efectos de su ordenamiento, preservación y sistematización, para proceder luego a su apertura en condiciones correctas y regulares, de acuerdo a reglas

\footnotetext{
${ }^{13}$ El sr. Luis María Paravís, de extensa y destacada actuación como funcionario parlamentario, fue secretario de la mencionada Comisión.

${ }^{14}$ Sobre la situación y los debates generados en torno a ambos archivos, en particular por la tramitación pública y la posterior publicación fragmentaria de la documentación contenida en ambos, varios historiadores del campo de la Historia Reciente han hecho públicos sus pareceres. Para visiones e información de ese debate cfr. WSCHEBOR PELLEGRINO, Isabel. "Detrás de los Berruti Papers" (5 de agosto de 2016); "Archivos sensibles son los que perturban a los poderosos" (17 de octubre de 2016); WSCHEBOR PELLEGRINO, Isabel y COLLAZO, Camilo, "Nuestras reservas del caso" (22 de agosto de 2016); DUFFAU, Nicolás, "Por qué sí, pero no asî́" (5 de agosto de 2016); MARKARIAN, Vania, "Brechaleaks: la culpa no es solo del chancho" (5 de agosto); LARROBLA, Carla, "Buenas prácticas" (5 de agosto de 2016); todos disponibles en https://ladiaria.com.uy. También debe consultarse la página on line de Brecha, en especial para consultar los fundamentos esgrimidos por el periodista Samuel Blixen y por la dirección del semanario para la publicación de un cúmulo importante de la documentación del llamado Archivo Berruti. Cfr. www.brecha.com.uy. También puede consultarse a propósito de estas últimas polémicas sobre el uso de los archivos represivos WSCHEBOR PELLEGRINO, Isabel, "Con archivos el pasado no es el mismo", en Anuario SERPAJ 2013-2014, Montevideo, SERPAJ, 2014, pp. 135 a 141; WSCHEBOR PELLEGRINO, Isabel, "Denunciar, debatir e instituir la verdad. Los ecos de Zelmar Michelini sobre los crímenes de la dictadura", en CAETANO, Gerardo (Coord.), Zelmar Michelini. Razones de una conducta. Acción y pensamiento. Montevideo, Planeta, 2017, pp. 301 a 314. ${ }^{15}$ Ibídem.
} 
generales que provengan de las leyes y reglamentos correspondientes. Para ello, en primer lugar, resulta imperativa una actividad proactiva del Estado en la búsqueda de esos archivos o de lo que resta de los mismos, en procura de documentación de decisiva relevancia, no sólo para los historiadores sino en particular para muchos procesos judiciales actualmente en curso. ${ }^{16}$ A nuestro juicio, tanto en el Uruguay como en otros países latinoamericanos, sobre el particular es mucho lo que resta por hacerse. Asimismo, en nuestra perspectiva de análisis radica aquí un factor muy relevante para la consolidación institucional de estrategias y políticas públicas orientadas a la previsión y a la no reiteración de procesos de violación de los derechos humanos.

En la compulsa que distintos investigadores han podido hacer en los archivos de la represión identificados y ubicados hasta el momento, ha podido confirmarse el carácter fuertemente burocrático del personal militar y civil adscrito durante el régimen dictatorial a este tipo de tareas. Los documentos iniciados en una dependencia aparecen frecuentemente reproducidos en multiplicidad de copias y enviados a otras secciones de los organismos de seguridad y oficinas del Estado sin motivo funcional claro en muchos casos. Esto ha permitido, por ejemplo, hallar documentos de los -hasta hoydesaparecidos archivos del Servicio de Inteligencia de Defensa (SID) y del Organismo Coordinador de Operaciones Antisubversivas (OCOA) en el Archivo de la Dirección Nacional de Información e Inteligencia (DNII), en el Archivo del Ministerio de Relaciones Exteriores, en dependencias del Ministerio de Defensa o en el domicilio particular de un militar fallecido, como en el caso ya señalado del coronel Elmar Castiglioni. Este tipo de circunstancias, verificadas en forma reiterada, fundamenta la necesidad de impulsar sin demora una amplia investigación sobre el paradero de los archivos de la represión en todas las dependencias públicas del Estado uruguayo, con especial atención a profundizar

\footnotetext{
${ }^{16}$ Para un debate a propósito de las tensiones implícitas y explícitas en el uso de los archivos represivos desde las perspectivas de los historias y de los agentes del Derecho en Uruguay, cfr. la polémica entre Vania Markarian y Alvaro Rico en Contemporánea. Historia y problemas del siglo XX. Año 7, volumen 7, 2016, pp. 177 a 210: "La situación de los archivos del pasado reciente".
} 
dicha búsqueda en las instalaciones de las Fuerzas Armadas, el Instituto Policial, el Ministerio de Defensa y el Ministerio del Interior. ${ }^{17}$

Entre los investigadores dedicados a estos temas existe convicción que en este plano hay mucho para buscar y también para encontrar. Esta convicción no surge de simples especulaciones sino de hechos muy concretos. Reseñemos algunos de ellos. Durante la primera administración del presidente Tabaré Vázquez, como ya se ha señalado, fue hallado en dependencias del Ministerio de Defensa Nacional un repositorio documental proveniente de la Escuela de Inteligencia del Ejército, el que en el año 2009 fue entregado al Archivo General de la Nación, que luego ordenó los documentos y de acuerdo a la legislación vigente en 2011 los puso en reserva por 15 años. ${ }^{18}$ Otro ejemplo especialmente significativo en esta dirección alcanzó publicidad en los últimos días del primer gobierno de Vázquez. En efecto, en febrero del 2010, a partir de una iniciativa tomada en tal sentido por el entonces Ministro de Defensa Nacional, Dr. Gonzalo Fernández, se informó públicamente que un grupo de técnicos archivólogos contratado por dicho Ministerio había logrado localizar en el Hospital Militar un archivo con aproximadamente 40.000 carpetas con historias clínicas de la década de 1970, entre las que presuntamente podrían encontrarse fichas de detenidos que en aquella época recibieron atención en dicho nosocomio. ${ }^{19}$ En esa misma dirección habría que referir el hallazgo de

\footnotetext{
17 Cfr. sobre esta particular: Relevamiento de archivos y repositorios documentales sobre derechos humanos en Uruguay. Montevideo, AGU-MEC-PNUD, 2007; y RICO (coord.), 2008.

18 Como ha explicado Vania Markarian: "Esto quiere decir que, por ese lapso, los mismos estarían disponibles para los directamente involucrados mediante pedido expreso de ellos, sus familiares o apoderados y permanecerían abiertos sin restricciones para la justicia y otras investigaciones sobre violaciones a los derechos humanos." Cfr. MARKARIAN, Vania, "Brechaleaks: la culpa no es solo del chancho”, ob. cit. Como se señalara a título expreso en la página web del AGN, en el año 2009 el Ministerio de Defensa Nacional hizo entrega a dicho Archivo de 11 cajas con rollos de microfilmes y su respaldo en 51 DVD, de material de archivo proveniente la referida Escuela de Inteligencia del Ejército. También fue entregado al AGN a fines de año 2008 material proveniente del Estado Mayor Conjunto (ESMACO), ocho cajas con documentación encontrada, que también se encuentra en proceso de clasificación. Cfr. www.agn.gub.uy.

${ }^{19}$ Cfr. La República, Montevideo, 26 de febrero de 2010, p. 2. "Hallaron archivo en el Hospital de las FFAA con historias clínicas". En dicha nota se recogen declaraciones del exministro y exsecretario de la Presidencia, Gonzalo Fernández, de decisiva actuación en el impulso de la nueva política de Tabaré Vázquez en torno a la investigación de los delitos de lesa humanidad cometidos durante la dictadura, en las que consigna entre otros conceptos: "Es notorio que cada vez que se ha pedido una historia clínica de algún caso vinculado a los derechos humanos, la historia no aparece. (...) Decidi hacer una investigación un poco más profunda (...) y hemos descubierto que hay en absoluto desorden una especia de depósito de historias viejas donde habrá de todo desde luego, pero a lo mejor aparecen algunas historias de detenidos y asistidos en el Hospital en aquella época (...). (Dicha investigación) obedece a
} 
documentación en el domicilio particular del coronel fallecido Elmar Castiglioni, en el marco de la causa judicial del homicidio de Fernando Miranda.

La identificación de este tipo de repositorios documentales durante los últimos años refuerza la hipótesis de trabajo antes señalada y alienta la expectativa de obtener más resultados positivos una vez que se sistematice y profundice este tipo de indagatorias. Una prueba suplementaria de esto ha sido el hallazgo en el Archivo General de la Nación de documentación vinculada con la acción del Partido Nacional durante la última dictadura, lo que motivó la entrega solemne de la misma en el año 2010 por parte del entonces presidente uruguayo José Mujica al entonces presidente del Directorio nacionalista, el expresidente Luis Alberto Lacalle. ${ }^{20}$

A partir del magro antecedente de los Informes solicitados a los comandantes de las tres armas en el año 2005, requiriéndoles información sobre el destino de los detenidos desaparecidos, los que fueron entregados cargados, en mayor o menor medida, de omisiones, ocultamientos y versiones erróneas, no parece que el camino más eficaz en esta dirección sea solamente reeditar solicitudes genéricas a los mandos. Si bien resulta importante el promover con firmeza nuevas órdenes para acometer y profundizar estas indagatorias por parte de las jerarquías civiles y militares, resulta imperativo sumar a las mismas otras investigaciones independientes, realizadas con todas las garantías del caso en forma paralela por personal idóneo, a partir de iniciativas formales de los Poderes Públicos y/o de instituciones civiles habilitadas específicamente por estos, con neto apoyo de las autoridades de gobierno y amplia legitimidad social.

En una dirección similar, resulta también necesario consolidar articulaciones sistemáticas e institucionalizadas, que permitan una consulta eficiente y ágil de los archivos represivos que han podido ubicarse en los países

una orden mía que quiere saber si hubo o no destrucción de historias y a ordenarlas un poco. No es una búsqueda de un caso especifico sino una búsqueda general”.

${ }^{20}$ Cfr. La República, Montevideo, 14 de mayo de 2010, p. 3. "Hallazgo. Fueron encontrados "por casualidad" en el Archivo General de la Nación. Mujica le entregó al Partido Nacional documentos sustraídos por la dictadura". Entre los documentos hallados figuraba la carta de renuncia de Wilson Ferreira Aldunate a su candidatura presidencial en 1984 y las actas de escrutinio de las elecciones de noviembre de 1971, impugnadas entonces por el P. Nacional. 
de la región, habida cuenta de la dinámica fuertemente transnacional que adoptaron las prácticas y operaciones de terrorismo de Estado durante las últimas dictaduras del Cono Sur. Si bien ya han podido concretarse ejemplos específicos en este sentido, incluso con algunas asociaciones de tipo institucional, ${ }^{21}$ la consolidación de los caminos de colaboración en esta dirección requieren una acción mucho más profunda y permanente. Se impone que sobre este particular, por ejemplo, los Estados parte del Mercosur acuerden las bases de una política pública de perfil regional, que permita investigar en esa misma clave con una eficacia supranacional que pueda corresponderse a la que exhibieron trágicamente los militares de las dictaduras conosureñas en ocasión de la "Operación Cóndor". ${ }^{22}$

Sin embargo, como se han encargado de destacar distintos investigadores, ha prevalecido en forma muy nítida en el continente, la dimensión nacional sobre la internacional o regional en lo que refiere a los distintos aspectos involucrados en los proceso de "justicia transicional", lo que entre cosas ha provocado fuertes asimetrías en cuanto a los avances logrados en estas materias en los distintos países.

\begin{abstract}
"Los logros -ha dicho sobre este particular Ana Buriano- han sido diversos en América Latina. Pese a que han ejercido presión interna y a la existencia de un marco jurídico internacional que acota cada vez más el encubrimiento de los crímenes impulsados desde el Estado (...), «el ámbito político de las prácticas de la memoria sigue siendo nacional, no posnacional o global.» (Huyssen, 2002). Así, las asimetrías en los avances logrados en cada país en torno al establecimiento de una memoria social sobre la violación de los derechos humanos en el pasado reciente guarda relación estrecha con las historias nacionales y las formas concretas en que se produjeron los tránsitos, los acuerdos de paz o los procesos democratizadores." (Buriano, 2009).
\end{abstract}

Sin la consolidación por parte de los Estados de políticas públicas comunes y supranacionales sobre estos temas, por ejemplo en el campo de la articulación en red de los archivos represivos identificados, seguirán reiterándose dificultades insalvables para la profundización de las investigaciones judiciales o históricas sobre el traumático pasado reciente de nuestros países. Pero para que haya políticas públicas regionales efectivas sobre

\footnotetext{
${ }^{21}$ Tal es el caso por ejemplo de convenios operativos entre Argentina y Uruguay para la utilización de documentación de los archivos existentes en el "Archivo de la Memoria" de la Secretaría de Derechos Humanos, radicada en la ciudad de Buenos Aires.

${ }^{22}$ Sobre este tema específico, cfr. MC SHERRY, J. Patrice, 2009; DUTRÉNIT, Silvia-VARELA PETITO, Gonzalo, 2010; entre otros.
} 
cualquier asunto, resulta imperativo que primero las haya y de manera consistente en el plano nacional de los Estados involucrados. ${ }^{23} \mathrm{Y}$ como se verá a continuación, el panorama sobre este particular resulta variado y en general deficitario.

\section{Entre el rigor profesional y un enfoque desde la perspectiva de los derechos: la situación de los archivos represivos hallados en Uruguay}

Si la identificación de los archivos represivos y la voluntad política para su acceso resultan definiciones primarias sin las cuales no hay avance posible, una efectiva utilización de los mismos, que pueda servir a los objetivos de la justicia y la verdad, plantea de inmediato otro tipo de exigencias en términos de políticas públicas: preservación, restauración, ordenamiento y clasificación, sistematización, normatización para un uso criterioso y ajustado de reglas universales para su acceso, entre otras tareas. Para que todo esto pueda incorporarse de veras en un sistema archivístico que se sustente en la perspectiva de los derechos, el rigor profesional con el que se actúe desde el comienzo resulta fundamental. La situación verificada en Uruguay respecto a este tipo de archivos, como también lo que podemos ir sabiendo de lo que ocurre al respecto en Argentina y Brasil, resulta confirmatoria de esta visión. ${ }^{24}$

En el Informe introductorio de la "Investigación Histórica sobre Detenidos Desaparecidos” en Uruguay antes referido, se hace una minuciosa descripción de los archivos relevados durante la investigación y de su situación actual, estableciéndose una serie de recomendaciones efectivas para una acción urgente y sistemática en esta dirección. Pero más allá de las irregularidades y deficiencias constatadas (presunta desaparición de archivos y repositorios documentales claves, dispersión y no sistematización de las fuentes existentes

\footnotetext{
${ }^{23}$ Sobre este particular, para citar un ejemplo, contrastan vivamente los avances concretados en la materia en Argentina respecto a una situación especialmente deficitaria y bloqueada exhibida por Brasil. Para una actualización de la situación de los archivos represivos en Brasil y Argentina, resulta muy útil e ilustrativo de las asimetrías mencionadas la lectura de los trabajos de Carlos Fico, "Archivos secretos de la dictadura brasileña" y de NAZAR, Mariana-PAK LINARES, Andrés, "El acceso a documentación relacionada con violaciones a los derechos humanos del último régimen militar en Argentina”, en Archivos y derechos humanos. Los casos de Argentina, Brasil y Uruguay. Montevideo, AGU, 2009, 56 pp.

${ }^{24}$ Ibídem.
} 
de acuerdo a criterios profesionales, penoso estado de preservación de materiales en varias dependencias, arbitrariedad o ausencia de pautas claras para su consulta pública en muchos casos, ausencia de digitalización y respaldo de buena parte de la documentación, en algunos casos falta de aplicación de criterios archivísticos profesionales, entre otros muchos problemas que podrían citarse), las premisas fundamentales de cualquier trabajo en la materia deberían orientarse en primer lugar a conceptuar la misma como una "cuestión de derechos", tanto individuales como colectivos. En esto, el conjunto de los países latinoamericanos, contra toda tentación de provincianismo, tiene que atender a la experiencia internacional en la materia y a los avances registrados en ese plano en la codificación de criterios estandarizados para operar sobre este tipo de documentación. En esa dirección resulta siempre indispensable legislar adecuadamente al respecto25, a lo que en forma simultánea debe sumársele la tarea de emprender sin demora una labor fuertemente profesional, con el asesoramiento directo de personas especialmente capacitadas para liderar una renovada política archivística de envergadura. En este sentido, cabe señalar que en el campo internacional, el fenómeno lamentable de la dictaduras y de los regímenes autoritarios del siglo XX ha generado indirectamente avances importantes en la definición de los criterios (políticos, jurídicos, historiográficos y archivísticos) para actuar sobre los archivos especialmente "sensibles" de los organismos represivos. 26

\footnotetext{
${ }^{25}$ Como se indica a título expreso algo más adelante, resulta imperioso "someter los archivos represivos a la ley". "Legislación y archivos -han señalado algunos de los principales expertos del Consejo Internacional de Archivos, en un Informe que se detalla a continuación- deben ir de la mano en los procesos de transición política. El legislador debe tener en cuenta los archivos y su papel instrumental para hacer que las medidas legislativas sean efectivas". Asimismo, la legislación a aprobar en este sentido, aunque ponga el acento en cómo proceder correctamente en la sistematización, ordenamiento y apertura pública de esos archivos, tendrá que llegar con posterioridad a la elaboración de normativa sobre aspectos conexos, al igual que lo que ocurre en otros países del mundo: formas de apoyo a demandas del Poder Judicial, compulsa de antecedentes sobre eventual participación en violaciones a los derechos humanos a propósito de personal adscrito a funciones de seguridad y vigilancia, etc. Para citar ejemplos concretos y cercanos a este respecto, puede citarse parte de la normativa que sobre este particular se encuentra vigente en la República Argentina y que refiere el necesario asesoramiento en este caso del "Archivo Nacional de la Memoria", dependiente de la Secretaría de Derechos Humanos de la Nación, en distintos temas: elaboración de informes sobre expedientes de ascensos y designaciones de oficiales en las FFAA; compulsas a propósito del personal que trabaja en tareas de servicio de vigilancia, custodia y seguridad de bienes y personas; lo mismo en relación al ingreso de personal en los organismos de seguridad del Estado o en los elencos docentes de los Institutos de formación dependientes de las FFAA; entre otros asuntos.

${ }^{26}$ En su libro precisamente titulado "Archivos y derechos humanos", Ramon Alberch I Fugueras enfatiza en los componentes de la accesibilidad y la desclasificación como las "llaves maestras" de un adecuado marco normativo para el tratamiento de los archivos represivos. A este respecto existe una amplísima y
} 
El tema de la legislación sobre archivos represivos reviste una importancia central y exige, además de ese enfoque primario fundado en el tema de los derechos, una sólida y consistente solución técnica. En la pasada legislatura uruguaya, en forma paralela a la adopción de una nueva política en materia de derechos humanos a propósito de lo ocurrido durante la última dictadura27, el Parlamento uruguayo aprobó un conjunto de leyes que configuran un primer marco normativo sobre la materia del que el país carecía hasta entonces. Aunque su estudio excede los límites de este artículo, se impone registrar las principales leyes aprobadas en esta dirección: i) la Ley 18.220, aprobada en el Parlamento y promulgada por el Poder Ejecutivo en diciembre del 2007, por la que se crea un Sistema Nacional de Archivos; ii) la Ley 18.331, aprobada y promulgada en agosto de 2008, referida al tema de la protección de datos personales; iii) la ley 18.381, dedicada al tema del acceso a la información pública, aprobada y promulgada en octubre de 2008; y iv) la ley 18.435, aprobada y promulgada en diciembre del 2008, para la creación de un Archivo Nacional de la Memoria. ${ }^{28}$

Salvo la última de las leyes referidas, las tres restantes, como ha señalado con acierto Vania Markarian, configuran un marco normativo que "no refiere específicamente a los archivos de la dictadura, a los producidos de tal año a tal año, en determinado contexto político o por determinada agencia. Estas leyes regulan la conservación y el acceso público de los documentos de archivo en general, estableciendo plazos precaucionales ...”29 De este modo, se trató de

muy reciente bibliografía a consultar. Para citar tan sólo dos ejemplos cercanos y de fácil acceso, entre otros muchos que podrían citarse, cfr. DA SILVA CATELA, Ludmila-JELIN, Elizabeth (comps.), Los archivos de la represión. Documentos, memoria y verdad. Madrid, Siglo XXI, 2002; y las publicaciones emanadas de las actividades de la Comisión Provincial por la Memoria, de la Provincia de Buenos Aires y con sede en La Plata, que contiene el archivo de la Dirección de Inteligencia la Policía de la Provincia de Buenos Aires (DIPBA).

27 Los principales cambios introducidos al respecto por el expresidentes Tabaré Vázquez durante su presidencia (2005-2010) fueron los siguientes: i) aplicación estricta de la Ley de Caducidad, lo que terminó "perforando" la interpretación abusiva del amparo de dicha norma por los gobiernos anteriores y habilitando el avance de investigaciones y procesos judiciales; ii) acción proactiva en la búsqueda de restos de los detenidos desaparecidos en cuarteles y otras dependencias, a partir de la información requerida a los mandos; iii) desarrollo entre el 2005 y el 2007 de la investigación histórica sobre el destino de los detenidos desaparecidos, en aplicación del artículo $4^{\circ}$ de la Ley de Caducidad, lo que dio lugar a la publicación de los cinco tomos ya citados (el último de los cuales fue dedicado a las investigaciones arqueológicas en la búsqueda de restos); iv) establecimiento de una comisión de seguimiento de estas actuaciones.

${ }^{28}$ Para ver el contenido de estas leyes así como el itinerario de todo su trámite parlamentario, consultar www.parlamento.gub.uy.

${ }^{29}$ MARKARIAN, 2009. 
evitar la creación de instituciones específicas vinculadas a la documentación represiva de la última dictadura, a los efectos de evitar varios inconvenientes que la experiencia y la doctrina internacionales han enfatizado respecto a que en la transferencia de documentos existe el riesgo de perder el vínculo con su contexto originario de producción.

Por el contrario, la última iniciativa referida, la Ley 18.435 de diciembre de 2008, derivada de un proyecto presentado desde la Dirección de Derechos Humanos del Ministerio de Educación y Cultura (y que contaba con el antecedente de un proyecto similar de autoría de la legisladora Beatriz Argimón) para la creación de un Instituto Nacional de Derechos Humanos, impulsa la creación de una institución específica con el cometido de

\begin{abstract}
"promover la importancia de la vigencia de los derechos humanos y de la democracia mediante el pleno ejercicio del derecho individual y colectivo a la verdad, a la memoria y al acceso a la información pública sobre las violaciones a los derechos humanos por parte del Estado, ocurridas en el período comprendido entre el 9 de febrero de 1973 y el $1^{\circ}$ de marzo de 1985. Se incorporarán, asimismo, documentos anteriores o posteriores a esas fechas, si a juicio del Consejo Directivo son considerados importantes para la recopilación de los materiales correspondientes al citado período." 30
\end{abstract}

La Ley consta de cuatro capítulos (Disposiciones Generales, Organización, Remisión, conservación y protección de los documentos, Disposiciones especiales $y$ transitorias) y dispone una serie de acciones plausibles en relación a los repositorios de los archivos represivos uruguayos. El Consejo Directivo de la nueva institución estará integrado por cinco miembros con voz y voto: los directores o directoras del Archivo General de la Nación, de la Biblioteca Nacional, del Museo Histórico Nacional y de la Dirección Nacional de Derechos Humanos del Ministerio de Educación y Cultura, a los que se sumará un representante de una organización defensora de los derechos humanos, designado por el Poder Ejecutivo. Sin embargo, a pesar de que la norma fue mejorada en relación al proyecto originalmente presentado, varios de sus contenidos han merecido objeciones, a nuestro juicio fundadas, en una carta

\footnotetext{
${ }^{30}$ Cfr. www.parlamento.gub.uy.
} 
promovida por un grupo de historiadores uruguayos en agosto de 2007, cuando el proyecto se encontraba en plena discusión parlamentaria. ${ }^{31}$

En esta nota, pese a reconocerse en forma expresa que esta iniciativa respondía "a una preocupación existente en varios sectores de la sociedad y en el seno del gobierno relacionada con la necesidad de investigar y dilucidar todos los episodios, contextos y procesos vinculados al ejercicio del terrorismo de Estado durante la última dictadura" y a valorarse "los avances que se han producido en este sentido gracias a las medidas adoptadas por el gobierno actual", se establecían también algunas críticas severas, en particular dirigidas a prevenir las consecuencias negativas del desmembramiento de los archivos existentes y de la selección discrecional de la documentación a incorporar en dicho archivo "al criterio de la Dirección" del nuevo organismo a crearse. En la mencionada nota se advertía:

\begin{abstract}
"creemos necesario decir que la propia creación del ANM [Archivo Nacional de la Memoria] atenta contra la integridad de los archivos al dejar librado «al criterio de la Dirección» la selección de los documentos que lo integrarán a partir del desmembramiento, transferencia o reproducción de los archivos existentes. Vale la pena recordar que el contexto institucional en que se producen los documentos es clave para interpretarlos. La mayoría de las leyes de archivos que rigen en otros países protege con especial celo la integridad de las series documentales".
\end{abstract}

La experiencia internacional en la materia coincide en la necesidad de la no dispersión de la documentación, en el imperativo de no separarla de su contexto de origen y en la recomendación de evitar la selección heurística discrecional en cualquier caso, habida cuenta de los muchos peligros que una acción en esos sentidos puede generar. Finalmente, este grupo de historiadores advertía en su misiva acerca de la abierta contradicción que este proyecto presentaba en relación con los otros proyectos sobre la materia que también entonces se encontraban en trámite parlamentario (y que como se ha anotado, fueron luego aprobados), en particular con el que estableció la creación de un sistema nacional de archivos (Ley 18.220):

"El Parlamento -se concluía en la carta- tiene en su agenda la creación de un sistema nacional de archivos (con media sanción de la Cámara de Senadores) y estudia la mejor forma de garantizar el acceso de todos los ciudadanos a la

\footnotetext{
31 Cfr. WSCHEBOR PELLEGRINO, Isabel, "Los documentos de archivo sobre la última dictadura uruguaya: quién accede y cómo", en Archivos y derechos humanos. Los casos de Argentina, Brasil y Uruguay." Montevideo, Archivo General de la Universidad de la República, 2009, pp. 41 y ss. En el Anexo 4 se publica en forma textual la carta aludida, con la referencia de sus firmantes (pp. 53 a 55).
} 
información pública. Ambos proyectos abordan los temas de los que venimos hablando de modo global y sistemático y ofrecen soluciones de largo plazo. Creemos que ése es el marco para pensar los asuntos relacionados con las memorias colectivas y públicas del período de la última dictadura. Es hora de asegurar y regular el derecho de los ciudadanos a acceder a los documentos de archivo en un contexto institucional integral, no fragmentado o amenazado por la discrecionalidad del administrador". 32

Aunque algunas de las críticas de la nota fueron en parte recogidas en la discusión parlamentaria (por ejemplo la mención explícita a que el material manejado por la nueva institución a crearse no sería en ningún caso documentación original sino copias autenticadas o la propia fórmula aprobada para la integración del Consejo Directivo), persisten algunas dudas respecto a la articulación efectiva de esta institución con varios de los principios establecidos por las otras leyes mencionadas del marco normativo aprobado.

A pesar del innegable avance logrado en la pasada legislatura con la aprobación de estas leyes, varios investigadores y archivistas han dejado expresa constancia acerca de la persistencia de preocupaciones y críticas. En su texto ya aludido, la historiadora Isabel Wschebor, luego de narrar los fuertes inconvenientes derivados de la consulta discrecional de muchos de esos archivos y de la ausencia de criterios sistemáticos para el acceso a los mismos, expresó también críticas fundadas respecto a otros puntos relevantes que persisten aun después de la aprobación de las leyes señaladas: sobreinterpretación de algunas disposiciones legales para negar informaciones a investigadores en algunas dependencias estatales, “zonas grises” en la delimitación precisa de lo que se entiende por "archivos sensibles" y por los requerimientos adecuados para su uso adecuado, ausencia de incentivos precisos para la adopción de nuevas políticas de archivo que ajusten los instrumentos más correctos de descripción y búsqueda, entre otros. 33

Por su parte, en otro texto reciente, la historiadora Vania Markarian profundizaba sobre el mismo particular, expresando su balance sobre la situación reciente en la materia. Luego de historiar la situación vivida en los últimos años y sus dificultades, a partir del discernimiento necesario entre el marco normativo aprobado y "las políticas sistemáticas que un Estado debe

\footnotetext{
32 Esta nota fue hecha pública el 24 de agosto de 2007, impulsada por un grupo de historiadores entre los que se encontraba el suscrito.

${ }^{33}$ Wschebor, 2009: pp. 42 a 44.
} 
tener para garantizar el acceso a sus documentos de archivo", Markarian ha enfatizado en el hecho que pese a los grandes avances verificados, a su juicio "se ha avanzado más en la apertura de algunos archivos para algunos usuarios que en la generación de políticas sistemáticas que cumplan con el marco normativo vigente." Luego de considerar que "la lucha por la apertura de los archivos debe llevarse adelante con cierta independencia de las condiciones políticas inmediatas", la investigadora concluye que en función de la realidad observada, a su juicio

\footnotetext{
"se va a tener que trabajar mucho para que el marco normativo se convierta en una realidad. (...) Hay que tener políticas de archivo que acompañen ese marco regulatorio y esto implica destinar recursos, porque será muy difícil cumplir con lo que allí se establece en las condiciones actuales. (...) Hay que tener decisión política y aplicar recursos para que se pueda dar satisfacción al espíritu de las leyes, que es sin lugar a dudas ampliar el acceso." 34
}

El seguimiento del caso uruguayo resulta entonces bien ilustrativo en el sentido del cúmulo de requerimientos políticos pero también técnicos para atender en la forma debida una temática de esta complejidad y sensibilidad social. Si bien el marco legal es crucial e indispensable, la adopción efectiva de políticas públicas adecuadas, que se correspondan con la normativa y que hagan viable la aplicación de las nuevas reglas establecidas, resulta también decisiva. Se trata en suma de un asunto cívico de dimensión central, sin duda prioritario en toda esa nueva institucionalidad ciudadana que debe acompañar el avance de los procesos de "justicia transicional".

\section{La experiencia internacional y sus enseñanzas}

Como se ha visto, la premisa para la rigurosidad de las estrategias a implementar para legislar y desplegar políticas apropiadas en torno a los archivos represivos y su apertura radica en actuar sobre estos temas como una "cuestión de derechos", tanto individuales como colectivos. Esta es la primera definición que emerge al relevarse la producción más relevante y reciente a nivel internacional sobre estudios y recomendaciones en la materia. Veamos a este respecto, en primer lugar, algunos fragmentos del texto final proveniente del "Proyecto UNESCO-Consejo Internacional de Archivos" titulado "Los Archivos de la Represión: Dimensión de un problema social que supera los límites de la

\footnotetext{
${ }^{34}$ Markarian, 2009.
} 
Archivística”, culminado y aprobado hace ya más de dos décadas, en 1995. El mismo reúne una serie de pautas y recomendaciones generales que consideramos muy pertinentes para su consideración específica en una etapa de consolidación y profundización de los trabajos sobre los archivos represivos en el marco de procesos de "justicia transicional." 35

En dicho texto, luego de realizar una serie de precisiones iniciales sobre la metodología empleada, el Grupo de Expertos realiza una consistente fundamentación acerca de la necesidad imperiosa y de las razones para priorizar en primer término la ubicación, identificación y conservación de los fondos documentales represivos. En el Informe de referencia se narran experiencias muy diversas sobre el particular, como los casos de Rodhesia o Grecia, en los que bajo circunstancias muy diversas se terminó destruyendo gran parte de los fondos documentales. Se alude también a los casos de España y de la ex República Democrática Alemana, en los que luego de arduas discusiones en distintos ámbitos institucionales y sociales, se optó por la conservación y sistematización de la documentación en instituciones especialmente dedicadas a ese destino (en muchos casos, como en el del Archivo de la Guerra Civil Española de Salamanca, a partir de la expropiación de los edificios que en los períodos dictatoriales habían sido sede de los organismos represores) ${ }^{36}$ y al cumplimiento de múltiples funciones derivadas de dicha responsabilidad. El Grupo de Expertos director de este proyecto adhirió firmemente a esta última opción y recomendó con especial énfasis que esa debía ser la base de las políticas a seguir en la materia, por razones que iban desde la consideración de la memoria o la investigación histórica, hasta el desarrollo de causas judiciales y derechos inalienables de las víctimas, de la sociedad y de la humanidad en su conjunto.

\footnotetext{
35 Ver Grupo ICA-UNESCO, "Los Archivos de la seguridad del Estado de los desaparecidos regímenes represivos", texto resumido por Antonio González Quintana del Informe aprobado por el Grupo de Expertos establecido por UNESCO y el Consejo Internacional de Archivos, compuesto por Antonio González Quintana (director del proyecto), Alejandro González Pobrete, Vladimir Kozlov, Narissa Ramdhani, Eliana Rezende Furtado de Mendoza, Mary Ronan, Dagmar Unvernhau, Lazlo Varga. Este Informe fue aprobado por este Grupo en una reunión celebrada en Salamanca en siembre de 1995.

${ }^{36}$ Otro caso similar al del Archivo de la Guerra Civil Española en Salamanca lo configura la Comisión Provincial por la Memoria de la ciudad de La Plata, que se encuentra alojado en la antigua sede de la Dirección de Inteligencia de la Policía de la Provincia de Buenos Aires (DIPBA).
} 
En esta última dirección, este Informe refería un conjunto de "derechos colectivos" y de "derechos individuales" que se consideraban como los ejes para diseñar "políticas de la memoria" consistentes ante los legados de pasados traumáticos signados por prácticas de Terrorismo de Estado. Entre esos derechos rectores a los que aludía el Informe de referencia merecen una especial consideración los siguientes:

\section{"Derechos Colectivos}

1. El derecho de pueblos y naciones a elegir su propia transición política está seriamente condicionado por la existencia de documentos. Sin archivos no hay plena libertad de opciones. (...)

2. El derecho a la integridad de la memoria escrita de los pueblos debe ser irrenunciable. (...) Las naciones tienen el derecho y la obligación de preservar su memoria, de la que es testimonio esencial la depositada en sus archivos. (...) El derecho a elegir la vía de transición excluye el de la destrucción de documentos.

3. El derecho a la verdad. Íntimamente ligado a los anteriores, los ciudadanos a través de su representación parlamentaria, o cualquier otra que sus legítimos representantes consideren adecuadamente representativa, tienen el derecho a una información global sobre la actuación del régimen anterior.

4. Derecho a conocer a los responsables de crímenes contra los derechos humanos. El derecho a conocer los nombres de los criminales y agentes de la represión debe ser considerado independientemente de cualquier decisión política sobre la exigencia o no de responsabilidades a los mismos, así como su posible continuidad como servidores públicos. (...) En democracia, los pueblos deben conocer el nombre de los responsables de tales atrocidades, evitándose su posible promoción política amparada en el anonimato de los crímenes contra los derechos humanos. (...)

\section{Derechos individuales}

1. Derecho a conocer el paradero de familiares desaparecidos en el periodo represivo. (...) Los archivos de la represión deben ser elemento fundamental para clarificar este extremo. (...)

2. Derecho al conocimiento de los datos existentes sobre cualquier persona en los archivos represivos, conocido como "habeas data". (...)

3. Derecho a la investigación histórica y científica. Todos los ciudadanos tienen el derecho a estudiar el pasado de la nación.

4. Derecho a la amnistía para presos y represaliados políticos. (...) Frecuentemente, sólo en los documentos de los archivos de los desaparecidos órganos represivos se hallan las pruebas de naturaleza política, religiosa, étnica o racial de los cargos judiciales o los despidos. (...)

5. $\quad$ Derecho a la compensación y reparación de daños sufridos por las víctimas de la represión. (...)

6. Derecho a la restitución de bienes confiscados (...)”. 37

A partir del señalamiento de estos derechos fundamentales en relación a la preservación de los archivos de la represión, el Grupo de Expertos antes

\footnotetext{
${ }^{37}$ Ibídem.
} 
mencionado enfatizó también sobre la necesidad, como ya hemos señalado, de "someter los archivos represivos a la ley". "Legislación y archivos -señalaron dichos expertos en su Informe- deben ir de la mano en los procesos de transición política. El legislador debe tener en cuenta los archivos y su papel instrumental para hacer que las medidas legislativas sean efectivas". A partir de esta convicción, la comisión emitió al final de su Informe cuatro recomendaciones finales:

\begin{abstract}
“1. Los conjuntos de documentos producidos o acumulados por los desaparecidos órganos de la represión deben ser puestos, tan pronto como sea posible, bajo el control de las nuevas autoridades democráticas, que deben proceder a censar tales conjuntos. (...)
\end{abstract}

2. Los documentos de los desaparecidos órganos represivos deben ser conservados en instituciones archivísticas enmarcadas en el sistema general de archivos de las administraciones públicas del país, o bien en instituciones archivísticas especialmente creadas por el nuevo régimen con el propósito de exigir responsabilidades, compensar a las víctimas de la represión o cualquier otro de los propósitos mencionados entre los derechos colectivos e individuales anteriormente relacionados. (...)

3. Deberán promoverse disposiciones que protejan legalmente los conjuntos documentales de los desaparecidos organismos represivos, como bienes de interés cultural. Si el país cuenta con leyes de protección de su patrimonio cultural, estos documentos deben situarse dentro de la categoría de bienes culturales protegidos. (...)

4. Deberán promoverse iniciativas legislativas encaminadas a garantizar el ejercicio de los derechos individuales con el apoyo de los archivos. (...) Deberá asimismo garantizarse el acceso a tal información de la forma más eficaz posible que garantice, a la vez, la privacidad de terceros. (Asimismo) toda persona que no haya estado al servicio de los órganos represivos, tendrá el derecho a determinar, una vez conocida la existencia de documentos sobre ella, si tales documentos pueden o no ser consultados por terceros, entendiéndose en todo caso, que sin declaración de los afectados, en un sentido u otro, los expedientes personales de las víctimas de la represión estarán cerrados a la consulta pública sin el permiso expreso de aquellos, o de sus herederos, por el período de tiempo que el legislador establezca." 38

Por último, en este Informe se establecía la necesidad de que los archivistas a cargo de los documentos de la represión asumieran en todos los casos su libre sometimiento a un código deontológico, que rigiera de manera precisa los límites y alcances de sus acciones profesionales. Como se observa, en las recomendaciones del Grupo de Expertos se enfatizaba en la necesidad de inscribir las nuevas orientaciones en relación a los archivos represivos dentro de "políticas de memoria" de amplio espectro, con soportes institucionales adecuados, con estrategias orientadas a preservar la memoria de las víctimas y a

${ }^{38}$ Ibídem. 
respaldar de múltiples formas y maneras la búsqueda de justicia, verdad y reparación. Del mismo modo, se dejaba también expresa constancia respecto a la necesidad imperiosa que los archivos, en el rediseño institucional de sus funciones y ordenamientos, asumieran como prioridad la cooperación con el ejercicio de los derechos individuales de los ciudadanos.

Por su parte, en 1997, el reconocido jurista francés Louis Joinet culminaba su famoso Informe (desde entonces conocido informalmente como Informe Joinet) sobre La Administración de la Justicia y los Derechos Humanos de los Detenidos. La cuestión de la impunidad de los autores de violaciones de los derechos humanos (civiles y políticos). Informe final elaborado por L. M. Joinet en aplicación de la decisión 1996/119 de la Subcomisión. Este documento fue elevado a la Comisión de Derechos Humanos de Naciones Unidas el 17 de abril de 1998 ( $52^{\text {a }}$ Reunión), la que lo incorporó en su resolución sobre la "Impunidad". 39

En su Informe, Joinet volvía a poner en el centro el tema de los derechos en las acciones a llevar a cabo sobre estas materias, al tiempo que exponía un conjunto de criterios coincidentes con los emanados del trabajo del grupo ICAUNESCO antes referido. En el primer apartado A, precisamente, enunciaba $E l$ derecho a saber, en el que desagregaba los temas de "las comisiones no judiciales de investigación" y la "preservación de los archivos relativos a las violaciones de los derechos humanos".

En relación a este último punto, señalaba Joinet:

\begin{abstract}
"Especialmente, luego de un proceso de transición, el derecho de saber implica que sean preservados los archivos. Las medidas que se deban tomar para esto tienen relación con los puntos siguientes: a) Medidas de protección y de represión contra la sustracción, la destrucción u ocultación; b) Realizar un inventario de archivos disponibles, que incluya los existentes en terceros países con la finalidad de que, con su cooperación, puedan ser consultados, o en su caso, restituidos; c) Adaptación a la nueva situación, de la reglamentación sobre el acceso a los archivos y su consulta, principalmente otorgando el derecho a toda persona que sea imputada la garantía de su derecho a respuesta y que éste sea incluido en su expediente." 40
\end{abstract}

\footnotetext{
${ }^{39}$ Para la consulta de este Informe puede confrontarse la página web de la UNESCO o también la del Foro por la Memoria: http: //www.nodo50.org/foroporlamemoria

${ }^{40}$ Ibidem, ítem 25 de la sección A, "El derecho a saber". Al final de su Informe, Joinet señala en forma textual: "iPara poder dar la vuelta a la página es necesario haberla leído! Pero la lucha contra la
} 
Por su parte, en el “Anexo II" del citado Informe, bajo el título de Conjunto de principios para la protección y la promoción de los Derechos Humanos, para la lucha contra la impunidad, Joinet señalaba las siguientes orientaciones en el apartado correspondiente a El derecho a saber:

\begin{abstract}
“A. Principios Generales
Principio 1. El derecho inalienable a la verdad. Cada pueblo tiene el derecho inalienable de conocer la verdad sobre los acontecimientos pasados, así como sobre las circunstancias y las razones que llevaron, por la violación masiva y sistemática de los derechos humanos, a la perpetración de crímenes aberrantes. El ejercicio pleno y efectivo del derecho a la verdad es esencial para evitar que en el futuro tales actos no se reproduzcan.
\end{abstract}

Principio 2. El deber de la memoria. El conocimiento por un pueblo de la historia de su opresión pertenece a su patrimonio y, como tal, debe ser preservado por medidas apropiadas en el nombre del deber a la memoria que incumbe al Estado. Esas medidas tienen por objeto la finalidad de preservar del olvido la memoria colectiva, principalmente para prevenir el desarrollo de tesis revisionistas y negacionistas.

Principio 3. El derecho de saber de las víctimas. Independientemente de toda acción de la Justicia, las familias de las víctimas tienen el derecho de conocer la verdad en lo que concierne a la suerte que fue reservada a sus parientes.

Principio 4. Garantías destinadas a hacer efectivo el derecho de saber. Corresponde a los Estados el tomar las medidas apropiadas para hacer efectivo el derecho de saber. Cuando las instituciones judiciales fallan, la prioridad debe ser, en una primera etapa, las medidas tendientes, por una parte, a la creación de comisiones no judiciales de investigación y, de otra parte, a la preservación y al acceso a los archivos". 41

En suma, Joinet entendía perfectamente la centralidad del tema de los archivos represivos para el éxito de los procesos de "justicia transicional". En la explicitación de algunos de los principios más relevantes para la promoción de los derechos humanos y para luchar contra la impunidad, el experto francés no casualmente insistía en la dimensión estratégica del tema de los archivos represivos, su identificación, preservación y acceso. En la defensa concreta del "derecho de saber de las víctimas", el tema de los archivos se constituía para él en una de las piedras angulares de un proceso de "justicia transicional" a la vez efectivo y legítimo.

impunidad no es tan solo una cuestión jurídica y política; ¿no nos olvidamos a menudo de su dimensión ética?".

${ }^{41}$ Ibídem. Anexo II. "Conjunto de principios para la protección y la promoción de los Derechos Humanos, para la lucha contra la impunidad". "I. El derecho a saber. A. Principios Generales." 


\section{Conclusiones}

Casi dos décadas después del Informe pionero del Grupo ICA-UNESCO que hemos presentado, el Director de aquel proyecto, Antonio González Quintana, realizó para la revista especializada Comma un trabajo de balance y perspectivas sobre la evolución reciente respecto a las actuaciones internacionales en el tema del tratamiento de los archivos de la represión. Luego de historiar los trabajos realizados sobre la materia a nivel de las distintas organizaciones internacionales de archivistas y de los órganos de la ONU especializados en el tema, González Quintana enfatizaba algunos elementos a su juicio rectores de los avances logrados. En sus palabras, se había logrado otorgarle "dimensión internacional" a esta problemática, se había podido formar convicción respecto a "la dimensión atemporal" del problema del seguimiento de los archivos de la represión, al tiempo que se había obtenido también en su opinión la "superación del marco de las fuentes meramente represivas en la defensa de los derechos humanos," accediéndose a una conciencia creciente acerca de la necesidad de llegar a lo que llamaba "los otros fondos sobre la represión”.

Afirmando sus dichos con ejemplos de acciones específicas llevadas adelante en distintas partes del mundo y con consideraciones técnicas de la disciplina archivística, González Quintana concluía su balance de lo ocurrido durante la última década destacando tres etapas significativas a su juicio de los nuevos enfoques dominantes en la materia: "El balance -decía- lo afrontamos desde los siguientes enfoques: 1) localización y conservación de fondos documentales; 2) nueva legislación sobre archivos en general o sobre archivos represivos en particular; 3) nuevas leyes e instituciones en relación con la gestión del pasado; y 4) nuevas instituciones archivísticas”. ${ }^{42}$

En el párrafo final de su artículo, González Quintana concluía:

"A modo de conclusión me gustaría señalar que dos son los retos principales de los archiveros en relación con los archivos de la represión: compromiso ético con los derechos humanos y compromiso deontológico con nuestra profesión. La evolución de los acontecimientos ha sido positiva en cuanto a la recuperación y apertura de archivos. El derecho internacional ha evolucionado, en el plano teórico sobre todo,

\footnotetext{
${ }^{42}$ Cfr. GONZÁlEZ QUINTANA, Antonio, 2004, pp. 59-74.
} 
pero también en la creación de instituciones como la Corte Penal Internacional, en coincidencia con nuestras propuestas. En este panorama nuestro papel debe situarse a la altura de las circunstancias, eso sí, con los paraguas preparados por si las nubes negras que la tensión internacional ha provocado en los últimos años descargan un fuerte aguacero sobre los Derechos Humanos”. 43

En la misma perspectiva de necesaria articulación entre el tema del tratamiento público de los archivos represivos como una cuestión de derechos se ha pronunciado en forma reciente Ramón Alberch I Fugueras:

\footnotetext{
"En el trasfondo de la necesidad de recuperar la memoria del pasado, lejano o reciente, existe la convicción de que es una realidad íntimamente ligada a los valores de verdad y justicia, que devienen ineludibles en los procesos de restitución de los derechos humanos y la convivencia democrática. (...) La memoria, en la medida en que deviene conocimiento, se convierte en una poderosa herramienta de recuperación de los derechos ciudadanos...” (ALBERCH, 2008)
}

¿Cómo se encuentran los países latinoamericanos de hoy respecto a estos temas especialmente sensibles de "derechos humanos", centrales en toda comunidad democrática? ¿Cuánta relevancia se le ha dado a este punto en los procesos de "justicia transicional" vividos y todavía en curso? ¿Cuán eficaz se ha sido en la redefinición institucional más perdurable en torno a "políticas de memoria" genuinamente democráticas? ¿Pueden abrigarse expectativas respecto a la adopción inmediata de criterios rigurosos en el tratamiento de los archivos represivos? ¿Son objeto estos últimos de políticas públicas, tanto nacionales como supranacionales, dirigidas a terminar de una vez con los obstáculos persistentes para la confirmación de las obligaciones estatales de investigar, sancionar y reparar? En definitiva, ¿se han asumido todos estos requerimientos como una cuestión radical de derechos humanos, con todas sus consecuencias inherentes?

Lamentablemente, la respuesta a estas preguntas, más allá de las asimetrías anotadas en los casos nacionales, no puede sino converger en la convicción de que nuestros países se encuentran muy lejos de haber consolidado un rumbo como el que se recomienda y practica en la experiencia internacional sobre los archivos represivos. En este como en tantos otros temas, nos hemos quedado rezagados en cuestiones que hacen a aspectos centrales del respeto a “derechos humanos" fundamentales. Y como tantas veces se ha dicho con razón, estos temas no involucran únicamente a especialistas sino que comprometen a

\footnotetext{
${ }^{43}$ Ibidem, p. 74.
} 
la ciudadanía en su conjunto, no pueden responderse a cabalidad desde perspectivas estrechamente nacionales sino que exigen compromisos e institucionalidad de carácter supranacional. Como se señalaba en el comienzo del texto, nuestro argumento central respecto al tema abordado apunta a que el mismo exige una conceptualización integral como una cuestión de derechos fundamentales. A la luz de lo ocurrido en los últimos años y de la experiencia regional e internacional, urge pues emprender con firmeza y decisión un conjunto de acciones impostergables en la materia. Cuanto más pase el tiempo más difícil será la tarea. En este tema, como en tantos otros, nos equivocamos cuando no incorporamos mundo en nuestras iniciativas cívicas. Aquí también radica un cimiento fundamental de toda convivencia democrática.

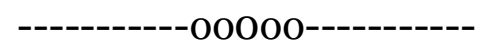

\section{Bibliografía}

AGUILAR FERNÁNDEZ, Paloma, (2008). Políticas de la memoria y memorias de la política. Madrid, Alianza Editorial.

ALBERCH I. FUGUERAS, Ramón, (2008). Archivos y derechos humanos. Gijón, Ediciones Trea.

AMBOS, Kai; MALARINO, Ezequiel; ELSNER, Gisela (eds), (2009). Justicia de transición con informes de América Latina, Alemania, Italia y España". Montevideo, Fundación Konrad Adenauer.

AUTORES VARIOS, (2009). Diccionario de Derechos Humanos. Cultura de los derechos en la era de la globalización. México, FLACSO.

BURIANO, Ana, (2009). "Memorias y derechos humanos en América Latina", en Diccionario de Derechos Humanos. Cultura de los derechos en la era de la globalización. México, FLACSO.

DA SILVA CATELA, Ludmila y JELIN, Elizabeth (comps.), (2002). Los archivos de la represión. Documentos, memoria y verdad. Madrid, Siglo XXI.

DECKER, Richard; KEPPLER, Elise, (2004). "Beyond the Hague: The Challenges of International Justice," en Human Rights Watch World Report, http://hrw.org.

DUTRÉNIT, Silvia; VARELA PETITO, Gonzalo, (2010). Tramitando el pasado. Violaciones de los derechos humanos y agendas gubernamentales en casos latinoamericanos. México, FLACSO-CLACSO.

ELSTER, Jon, (2006). Rendición de cuentas. La justicia transicional en perspectiva histórica. Buenos Aires, Katz.

FICO, Carlos, (2009). "Archivos secretos de la dictadura brasileña", en Wschebor; Markarian (eds), Archivos y derechos humanos. Los casos de Argentina, Brasil y Uruguay. Montevideo, AGU. 
GONZÁLEZ QUINTANA, Antonio, (2004). "Los archivos de la represión: balance y perspectivas", Comma, 2004-2, pp. 59 a 74 .

ICA-UNESCO, (2005). "Los Archivos de la seguridad del Estado de los desaparecidos regímenes represivos.” Salamanca, ICA-UNESCO. (Ha sido publicado también en las revistas Janus, Comma y Anabad, en distintos idiomas.)

JOINET, Louis, (1996). La Administración de la Justicia y los Derechos Humanos de los Detenidos. La cuestión de la impunidad de los autores de violaciones de los derechos humanos (civiles y políticos). Informe final elaborado por L. M. Joinet en aplicación de la decisión 1996/119 de la Subcomisión. Foro por la Memoria: http: //www.nodo50.org/foroporlamemoria.

HUYSSEN, Andreas, (2002). En busca del futuro perdido: cultura y memoria en tiempos de globalización. México, FCE - Goethe Institut.

LUTZ, Ellen - SIKKINK, Kathryn, (2000). "International Human Rights Law and Practice in latin America", International Organization, 54, pp. 633659.

MARKARIAN, Vania, (2009). "El uso de archivos sensibles y su debate actual" en Memoria, dictadura y derechos humanos: Ponencias del curso realizado entre el 10 y el 31 de agosto de 2009, Montevideo: $\mathrm{AEBU} / \mathrm{MEC}$.

MARKARIAN, Vania; WSCHEBOR, Isabel, (2007). Relevamiento de archivos y repositorios documentales sobre derechos humanos en Uruguay. Montevideo, AGU-MEC-PNUD.

MC SHERRY, J. Patrice, (2009). Los Estados depredadores: la Operación Cóndor y la guerra encubierta en América Latina. Montevideo, LOM Ediciones-Ediciones de la Banda Oriental.

NAZAR, Mariana; PAK LINARES, Andrés, (2009). "El acceso a documentación relacionada con violaciones a los derechos humanos del último régimen militar en Argentina", en Wschebor-Markarian (eds), Archivos y derechos humanos. Los casos de Argentina, Brasil y Uruguay. Montevideo, AGU.

RICO, Álvaro (coord.), (2007). Investigación Histórica sobre Detenidos Desaparecidos. 5 Tomos. Montevideo, IMPO.

RICO, Álvaro (coord.), (2008). Investigación histórica sobre la dictadura y el terrorismo de Estado en el Uruguay (1973-1985). Montevideo, Ediciones de la Cruz-CEIU.

ROTTBERG, Angelika, (2005). Entre el perdón y el paredón. Preguntas y dilemas de la justicia transicional. Bogotá, Ediciones Uniandes/IDRC.

SIKKINK, Kathryn, (2005). "Patterns of Dynamic Multilevel Governance and the Insider-Outsider Coalition", en Donatella Della Porta y Sidney Tarrow (eds.), Transnational Protest and Global Activism, Nueva York, Rowman and Littlefield, pp. 151-173.

SIKKINK, Kathryn; BOOTH-WALLING, Carrie, (2008). "La cascada de justicia y el impacto de los juicios de derechos humanos en América Latina”, en 
Cuadernos del CLAEH, 96-97, Montevideo, CLAEH, pp. 15-40.

TEITEL, Ruti G. (2003), Genealogía de la Justicia Transicional, http://www.publicacionescdh.uchile.cl

VINJAMURI, Leslie; SNYDER, Jack, (2004). "Advocacy and Scholarship in the Study of International War Crimes Tribunals and Transitional Justice", Annual Review of Political Science, 7, mayo, pp. 345-362.

WSCHEBOR PELLEGRINO, Isabel, (2009). "Los documentos de archivo sobre la última dictadura uruguaya: quién accede y cómo", en Wschebor; Markarian (eds.), Archivos y derechos humanos. Los casos de Argentina, Brasil y Uruguay. Montevideo, AGU. 